\begin{tabular}{|c|c|}
\hline Title & Fast computation of copper and iron losses using model order reduction \\
\hline Author(s) & Hiruma, Shingo; Igarashi, Hajime \\
\hline Citation & $\begin{array}{l}\text { International journal of applied electromagnetics and mechanics, 60(S1), S79-S86 } \\
\text { https://doi.org/10.3233/AA E-191107 }\end{array}$ \\
\hline Issue Date & 2019-05-30 \\
\hline DOC URL & http:/hdl.handle.net/2115/75088 \\
\hline Rights & The final publication is available at IOS Press through http://dx.doi.org/10.3233/JA E-191107 \\
\hline Tyре & article (author version) \\
\hline File Information & hiruma_final.pdf \\
\hline
\end{tabular}

Instructions for use 


\title{
Fast Computation of Copper and Iron Losses Using Model Order Reduction
}

\author{
Shingo HIRUMA*, and Hajime IGARASHI* \\ * Graduate School of Information Science and Technology, Hokkaido University, Hokkaido, Japan \\ E-mail: hiruma@em.ist.hokudai.ac.jp
}

\begin{abstract}
This paper introduces a new method for fast computing of the iron and copper losses in electromagnetic systems. In the method, the finite element equation is reduced to the equivalent Cauer circuit via model order reduction. The nonlinear property due to core saturation is pre-computed and included in the Cauer circuit. While the copper loss is computed as the Jule loss in the circuit, the iron loss is computed in the post process by restoring the field distribution from the solution to the circuit equation with aid of the proper orthogonal decomposition.
\end{abstract}

Keywords: Cauer circuit, eddy current problems, equivalent circuit, proper orthogonal decomposition.

\section{INTRODUCTION}

Recently, the model order reduction (MOR) techniques have attracted attentions because it can greatly reduce computational cost and memory usage. It has been shown that the MOR techniques allows us to reduce the Maxwell equations to the rational function [1] and also the continued function that is equivalently represented by the Cauer circuit [2]-[4]. While the copper losses obtained from the equivalent Cauer circuit have been shown very accurate, it has been difficult to accurately evaluate the iron losses in the magnetic core which include the hysteresis and eddy current losses from the circuit analysis. We here introduce a new method to restore the magnetic field from the solution to the circuit equation for computing the iron losses. In the proposed method, the Cauer circuit is built directly from the discretized Maxwell equations. The nonlinearity due to the core saturation is considered in the Cauer circuit by replacing the DC magnetic field with that obtained from the proper orthogonal decomposition (POD)[5]-[8]. The iron losses are here computed in the post process by applying the Steinmetz [9] equation to the restored field although other loss-evaluation methods can also be used.

\section{Linear problem}

\section{FORMULATION}

First, we consider a linear eddy current problem which is governed by the quasi-static Maxwell equation

$$
\operatorname{rot} v \operatorname{rot} \boldsymbol{A}+\mathrm{j} \omega \sigma \boldsymbol{A}=\boldsymbol{J}
$$

where $\boldsymbol{A}, \boldsymbol{J}, \boldsymbol{v}, \mathrm{j}, \omega, \sigma$ are the vector potential, external current density, magnetic reluctivity, imaginary unit, angular frequency, and conductivity, respectively. In the field analysis, it is assumed that the eddy currents exist only in the coil windings so that the iron losses are considered in the post process. The equation is discretized by the finite element method (FEM) to obtain

$$
(\mathrm{K}+s \mathrm{~N}) \boldsymbol{x}=\boldsymbol{b} I
$$

where $\mathrm{K}, \mathrm{N} \in \mathbb{R}^{n \times n}, \boldsymbol{b} \in \mathbb{R}^{n}, \boldsymbol{x} \in \mathbb{C}^{n}, I, s$ are the FE matrices, source and unknown vectors, external current, and complex frequency, respectively. The terminal voltage $V$ of the coil is given as 


$$
V=s \int_{\Omega_{\mathrm{c}}} \boldsymbol{A} \cdot \boldsymbol{j} d \Omega+R_{\mathrm{DC}} I=s \boldsymbol{b}^{\mathrm{T}} \boldsymbol{x}+R_{\mathrm{DC}} I
$$

where $\Omega_{\mathrm{C}}, \boldsymbol{j}, R_{\mathrm{DC}}$ denote the coil region, the unit vector parallel to the coil windings, and the DC resistance, respectively. By eliminating $\boldsymbol{x}$ from (3), we have the input impedance of the system as

$$
Z=V / I=s \boldsymbol{b}^{\mathrm{T}}(\mathrm{K}+s \mathrm{~N})^{-1} \boldsymbol{b}+R_{\mathrm{DC}}
$$

By applying the continued fraction via the Lanczos process (CVL) method [4] to the transfer function in equation (4), $Z$ can be written in the form of a continued function as

$$
Z=R_{\mathrm{DC}}+\frac{1}{\frac{1}{s \kappa_{0}}+\frac{1}{\frac{1}{\kappa_{1}}+\frac{1}{\frac{1}{s \kappa_{2}}+\frac{1}{\frac{1}{\kappa_{3}}+\frac{1}{\frac{1}{s \kappa_{2 q-2}}+\frac{1}{\frac{1}{\kappa_{2 q-1}}}}}}}}
$$

The continued function (5) is found to be the input impedance of the Cauer circuit shown in Fig.1. Although the Cauer circuit can be obtained by PVL [2] and CLN [3] in a similar way, CVL has the advantages that the continued function is derived directly for the general linear systems. The field $\boldsymbol{x}$ in equation (2) can be restored from the solution to the circuit equation as

$$
\boldsymbol{x}=i_{0} \boldsymbol{u}_{0}+i_{1} \boldsymbol{u}_{1}+\cdots+i_{q-1} \boldsymbol{u}_{q-1}
$$

where $i_{0}, i_{1}, \cdots, i_{q-1}$ are the currents in the Cauer circuit and $\boldsymbol{u}_{0}, \boldsymbol{u}_{1}, \cdots, \boldsymbol{u}_{q-1}$ are the bases of the Krylov subspace $\mathcal{K}_{q}\left(\mathrm{~K}^{-1} \mathrm{~N}, \boldsymbol{b}\right)$ which are obtained in the CVL method. The first and higher terms in equation (6) represent the magnetostatic field and response fields due to the eddy currents because $i_{k}, k \geq 1$ vanish faster than $i_{0}$ in the low frequency limit. Based on this physical interpretation, the nonlinearity is introduced in the first stage of the Cauer circuit. The iron losses can be computed from the restored field (6) by using the loss-evaluation methods such as Steinmetz equation.

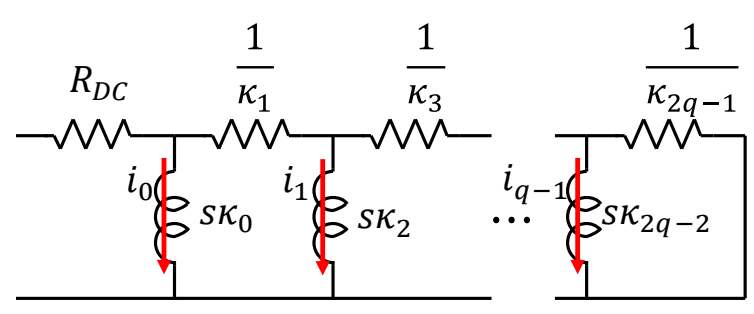

Fig. 1 Equivalent Cauer circuit

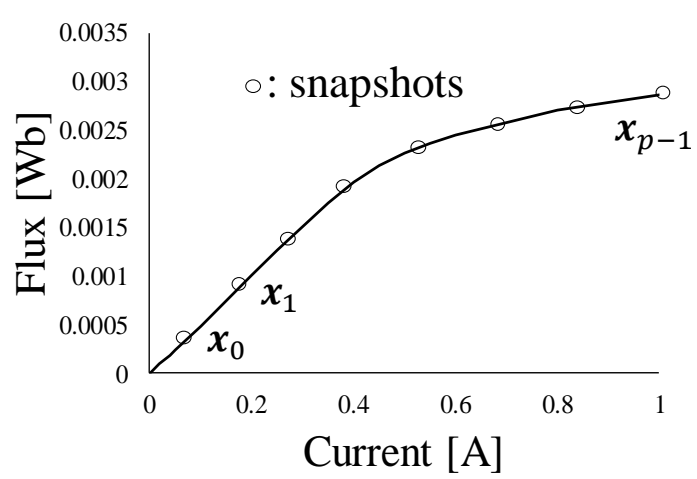

Fig.2 Current-flux characteristic 


\section{Nonlinear problem}

When we consider electric machines, it is important to consider the nonlinear property due to the core saturation. The nonlinearity is efficiently considered in the Cauer circuit by introducing the nonlinear current-flux characteristic which is shown in Fig.2 to the first stage of the Cauer circuit [2][10][11]. The nonlinear characteristic is obtained by solving the FE equation derived from the static Maxwell equation for different external currents $I$. To evaluate the iron loss in the post process, we set up the following two hypotheses. The first hypothesis is that the magnetostatic field is dominant compared with the response fields, that is

$$
\left|i_{0} \boldsymbol{u}_{0}\right| \gg\left|i_{1} \boldsymbol{u}_{1}+\cdots+i_{q-1} \boldsymbol{u}_{q-1}\right|
$$

Based on this hypothesis, the magnetic nonlinearity is represented by the first stage of the Cauer circuit. The second hypothesis is that the core saturation has little influence on the circuit parameters $\kappa_{1}, \kappa_{2}, \cdots, \kappa_{2 q-1}$ which represent the response fields. The second hypothesis is valid when the magnetic flux interlinked with the coil has a negligible change due to the core saturation.

The field restoration (2) is not valid when the core saturation becomes strong because the first term $i_{0} \boldsymbol{u}_{0}$ which represents the magnetostatic field would have major change. In this paper, we employ POD [5]-[8] to obtain the magnetostatic field considering the magnetic saturation. To evaluate the current-flux characteristic shown in Fig. 2 , the static fields $\boldsymbol{x}_{0}, \boldsymbol{x}_{1}, \cdots, \boldsymbol{x}_{p-1}$ are computed using FEM where $p$ denotes the number of snapshots. The data matrix $\mathrm{X}=\left[\begin{array}{llll}\boldsymbol{x}_{\mathbf{0}} & \boldsymbol{x}_{1} & \cdots & \boldsymbol{x}_{p-1}\end{array}\right]$ is then decomposed by the singular value decomposition as

$$
\mathrm{X}=\left[\begin{array}{llll}
x_{0} & x_{1} & \cdots & x_{p-1}
\end{array}\right]=\mathrm{W} \Sigma \mathrm{V}
$$

Using the matrix $\mathrm{W}$, the nonlinear FE equation derived from the static Maxwell equation is reduced to

$$
\mathrm{W}^{\mathrm{T}} \mathrm{K}\left(\boldsymbol{x}_{r}\right) \mathrm{W} \boldsymbol{x}_{r}=\mathrm{W}^{\mathrm{T}} \boldsymbol{b} \mathrm{I}
$$

where $\boldsymbol{x}_{r} \in \mathbb{R}^{p}$ is the reduced unknown vector. We can obtain the magnetostatic field considering the core saturation by solving equation (9) using the Newton-Raphson (NR) method. In the post process, we restore the magnetic fields by solving (9) for the external current $I=i_{0}$ which is obtained from the analysis of the Cauer circuit. As a result, the restored field is now expressed as

$$
\boldsymbol{x}=\mathrm{W} \boldsymbol{x}_{r}+i_{1} \boldsymbol{u}_{1}+\cdots+i_{q-1} \boldsymbol{u}_{q-1}
$$

The proposed method is composed of two steps as shown in Fig.3. In the first step, the nonlinear equivalent Cauer circuit is analyzed at each time step to obtain the copper losses. Then, reduced nonlinear FE equation (9) is solved to obtain the magnetostatic fields considering core saturation, and we restore the magnetic fields from which we compute the iron losses in the post process.

\section{NUMERICAL RESULTS}

\section{Application to reactor}

To verify the proposed method, we apply the method to the analysis of an axisymmetric reactor which is shown in Fig. 5. The model is composed of a ferrite core PC47, and copper wires. The conductivity of the wire is $\mathbf{5 . 7 6} \times \mathbf{1 0}^{7}[\mathbf{S} / \mathbf{m}]$, and the radius is $\mathbf{1 . 7 5} \times \mathbf{1 0}^{-4}[\mathrm{~m}]$. DC resistance of the coil winding $\boldsymbol{R}_{\mathrm{DC}}$ is $\mathbf{0 . 6 7 9}[\boldsymbol{\Omega}]$. 


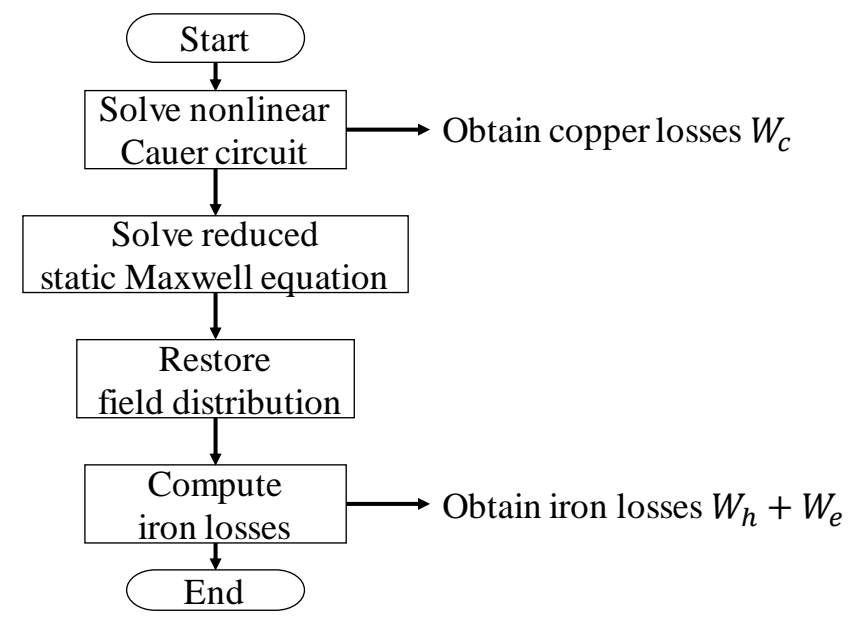

Fig. 3. The entire process to obtain the copper and iron losses.

First, we built the equivalent Cauer circuit of the reactor. The relative permeability of the magnetic core is assumed to be 6000 which is the initial permeability of the ferrite PC47. The circuit parameters are summarized in Table 1.

Then, we compute the current flux characteristic of the reactor by solving the static Maxwell equation. The number of snapshots $\boldsymbol{p}$ is fixed at 20. The resultant current flux characteristic is shown in Fig.5. We can see the inductance becomes small when the external current becomes large. By replacing $\boldsymbol{\kappa}_{\mathbf{0}} \mathbf{d} \boldsymbol{i}_{\mathbf{0}} / \mathbf{d} \boldsymbol{t}$ with $\mathbf{d} \boldsymbol{\Phi}\left(\boldsymbol{i}_{\mathbf{0}}\right) / \mathbf{d} \boldsymbol{t}$, we can obtain the Cauer circuit in time domain which is used in the nonlinear analysis.

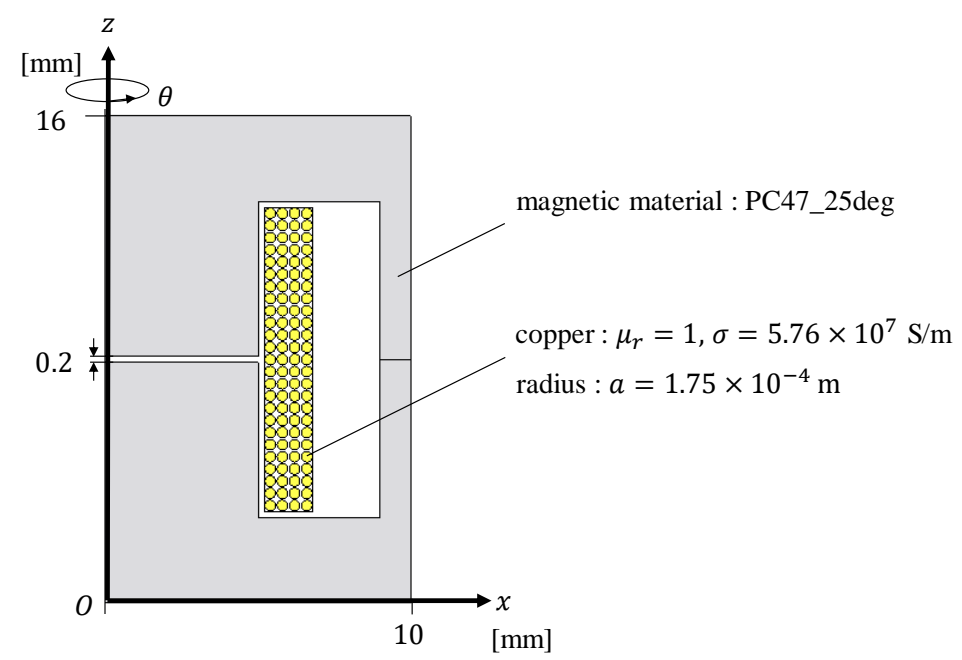

Fig. 4. Axisymmetric reactor

Table 1: Circuit parameters of reactor model

\begin{tabular}{ccc}
\hline$n$ & $\kappa_{2 n}$ & $\kappa_{2 n+1}$ \\
\hline 0 & $5.23 \times 10^{-3}$ & $2.28 \times 10^{-6}$ \\
1 & $1.95 \times 10^{-1}$ & $1.02 \times 10^{-7}$ \\
2 & $1.81 \times 10^{0}$ & $8.47 \times 10^{-8}$ \\
3 & $2.00 \times 10^{0}$ & $3.06 \times 10^{-8}$ \\
\hline
\end{tabular}




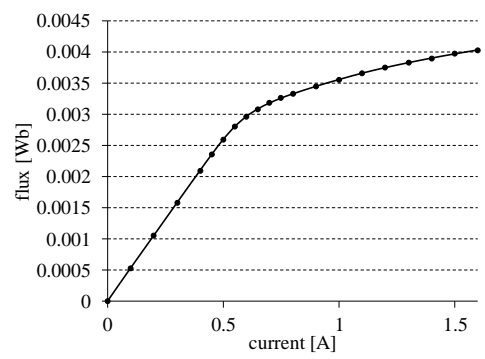

Fig. 5. Current flux characteristic of reactor

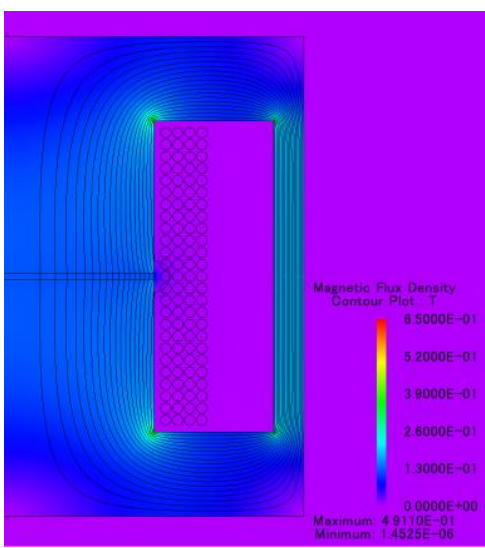

(a) $i_{0}=0.2[\mathrm{~A}]$

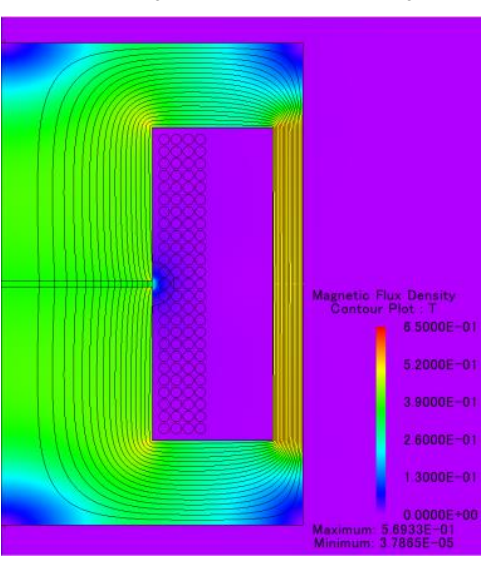

(b) $i_{0}=0.7[\mathrm{~A}]$



(c) $i_{0}=1.6[\mathrm{~A}]$

Fig. 6. Contour plot of magnitude of magnetic flux density and flux lines of reactor

Fig. 6 shows that the contour plot of the magnitude of the magnetic flux density and flux lines of the reactor when the external currents are $0.2,0.7,1.6[\mathrm{~A}]$. We can see that the distribution changes drastically depending on the external current due to the magnetic core saturation. This is the reason why the nonlinearity due to the saturation should be considered in the loss computation.

We assume a sinusoidal input voltage, $25 \mathrm{kHz}, 50 \mathrm{~V}$, as the power supply to the reactor and to the Cauer circuit. The instantaneous power of the Cauer circuit is plotted in Fig. 7. The responses in the transient and steady states computed by FEM are also plotted in Fig.7. We can see the results obtained by the Cauer circuit are in good agreement with those obtained by FEM. It takes about 11 minutes 25 second to build the nonlinear Cauer circuit and takes about 16 second to obtain the waveform shown in Fig. 7 (CPU: Intel Core i7-7700@3.6GHz, RAM: $8.00 \mathrm{~GB}$ ) whereas it would take at least 10 days to obtain the result by FEM under the same environment. The copper loss in steady state is evaluated to be

$$
W_{c}=\frac{1}{T} \int_{t_{1}}^{t_{2}} p(t) d t=4.18 \times 10^{-3}[\mathrm{~W}]
$$

The reduced FE equation (9) is solved for the peak current $i_{0}=6.00 \times 10^{-2}[\mathrm{~A}]$. The iron losses are here estimated from the Steinmetz equation. The result is

$$
W_{i}=W_{h}+W_{e}=1.06 \times 10^{2} f B_{m}^{2}+5.76 \times 10^{-4}\left(f B_{m}\right)^{2} .
$$

Since the peak current $i_{0}$ is not so large that the core is not strongly saturated, the resultant losses are not very different from those computed from the linear analysis of FEM. Total losses obtained by the proposed method and FEM are summarized in Fig.8. The relative errors in $W_{c}, W_{h}, W_{e}$ are $1.5,4.1$, and $4.1 \%$, respectively. The 


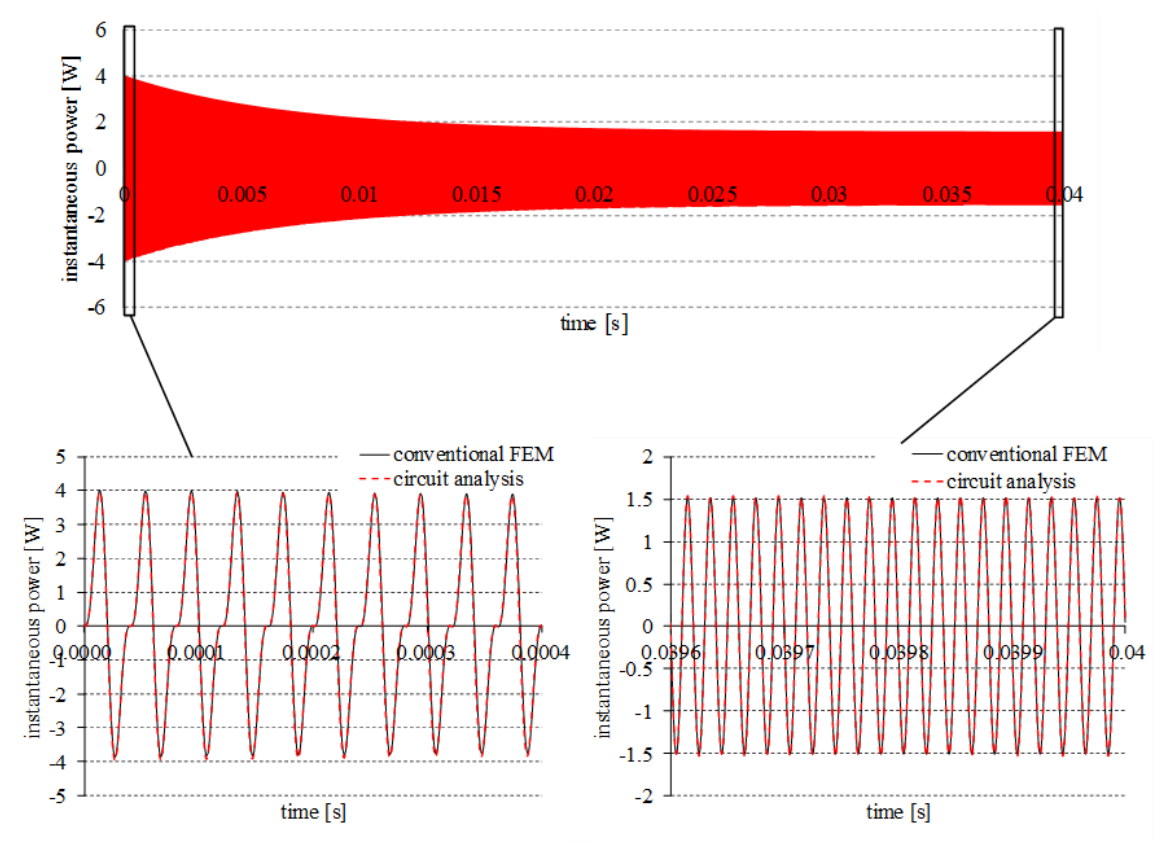

Fig. 7. Transient analysis of Cauer circuit with power supply of $25 \mathrm{kHz}, 50 \mathrm{~V}$ sinusoidal waveform

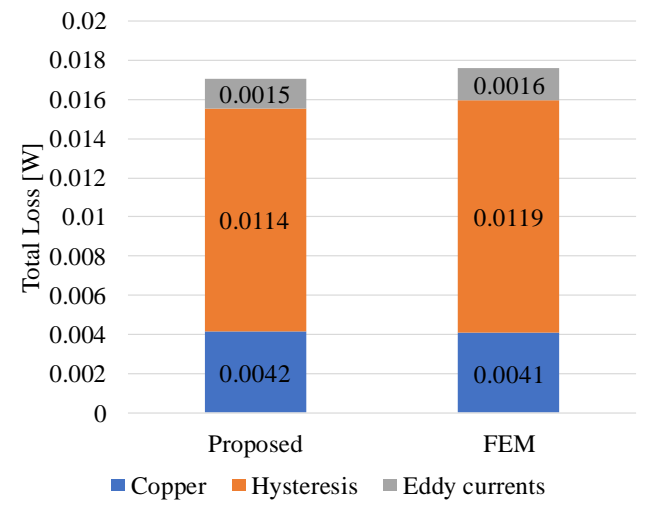

Fig. 8. Total losses obtained by proposed method (left) and FEM (right).

accuracy of iron losses depends on the accuracy of field restoration (10). The accuracy could be made higher when we increase the number of snapshots in POD or employ other MOR method for nonlinear field computations.

\section{CONCLUSIONS}

In this paper, we have proposed a new method to evaluate the copper and iron losses through the analysis of the Cauer circuit which is a reduced physical model of an electric machine. In the method, the copper losses are directly evaluated from the nonlinear Cauer circuit considering the core saturation. On the other hand, to evaluate the iron losses, the magnetic field is restored from the solution to the Cauer circuit and reduced Maxwell equation obtained by POD.

We have applied the proposed method to the axisymmetric reactor model for the validation of the method. The relative errors in the obtained copper and iron losses are 1.5 and $4.1 \%$. The accuracy of the iron losses depends on the accuracy of the MOR method.

We can perform fast transient computations for arbitrary inputs because analysis of the Cauer circuit needs much smaller computational cost compared to the original FE equation. When we consider the PWM excitation as a 
power supply, it is important to consider the hysteresis losses due to the minor loops. In this case, we have to employ the methods such as Preisach [12] and play [13] models which can include the losses from the minor loops. The transient loss analysis is remained for our future work.

\section{REFERENCES}

[1] P. Feldmann and R. A. Freund: "Efficient Linear Circuit Analysis by Padé Approximation via Lanczos Process," IEEE Transactions on Computer-Aided Design, Vol. 14, No. 5, p. 639-649, 1995.

[2] Y. Sato, T. Shimotani, and H. Igarashi, "Synthesis of Cauer Equivalent Circuit Based on Model Order Reduction Considering Nonlinear Magnetic Property," IEEE Transactions on Magnetics, vol. 53, no. 6, pp. 1-4, June 2017.

[3] A. Kameari, H. Ebrahimi, K. Sugahara, Y. Shindo, and T. Matsuo, "Cauer Ladder Network Representation of Eddy Current Fields for Model Order Reduction Using Finite-Element Method,” IEEE Transactions on Magnetics, vol. 54, no. 3, pp. 1-4, March 2018.

[4] S. Hiruma, H. Igarashi, "Synthesis of Cauer Circuit by Continued fraction via Lanczos Process," to be submitted.

[5] A. Chatterjee, “An Introduction to The Proper Orthogonal Decomposition,” Current Science, vol. 78, no. 7, pp. 808-817, 2000.

[6] D. Schmidthausler and M. Clemens, "Low-Order Electroquasistatic Field Simulations Based on Proper Orthogonal Decomposition," in IEEE Transactions on Magnetics, vol. 48, no. 2, pp. 567-570, Feb. 2012.

[7] Y. Sato and H. Igarashi, "Model Reduction of Three-Dimensional Eddy Current Problems Based on the Method of Snapshots, " in IEEE Transactions on Magnetics, vol. 49, no. 5, pp. 1697-1700, May 2013.

[8] T. Henneron and S. Clénet, "Model Order Reduction of Non-Linear Magnetostatic Problems Based on POD and DEI Methods, " in IEEE Transactions on Magnetics, vol. 50, no. 2, pp. 33-36, Feb. 2014, Art no. 7000604.

[9] C. P. Steinmetz, "On the law of hysteresis, " in Proceedings of the IEEE, vol. 72, no. 2, pp. 197-221, Feb. 1984.

[10] E. J. Tarasiewicz, A. S. Morched, A. Narang and E. P. Dick, "Frequency Dependent Eddy Current Models for Nonlinear Iron Cores," in IEEE Transactions on Power Systems, vol. 8, no. 2, pp. 588-597, May 1993.

[11] T. Miyazaki, T. Mifune, T. Matsuo, Y. Shindo, Y. Takahashi and K. Fujiwara, "Equivalent Circuit Modeling of Dynamic Hysteretic Property of Silicon Steel Under Pulse Width Modulation Excitation,” Journal of Applied Physics, 117, 17D110, 2015.

[12] F. Preisach, “Über die magnetische Nachwirkung," Zeitschrift für Physik, vol. 94, issue 5-6, pp. 277-302, May 1935.

[13] M. Brokate, "Some mathematical properties of the Preisach model for hysteresis, " in IEEE Transactions on Magnetics, vol. 25, no. 4, pp. 2922-2924, July 1989. 